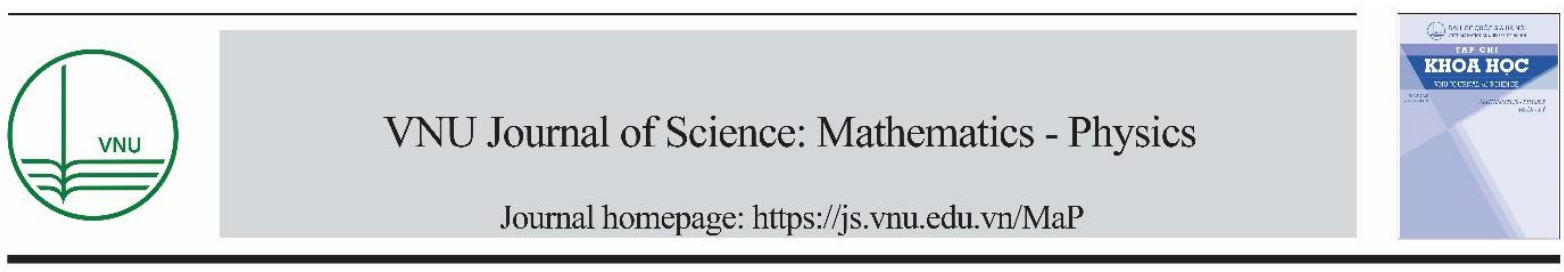

\title{
Original Article A Comparison Theorem for Stability of Linear Stochastic Implicit Difference Equations of Index-1
}

\author{
Nguyen Hong Son ${ }^{1,2^{*}}$, Ninh Thi Thu ${ }^{1}$ \\ ${ }^{1}$ Faculty of Mathematics, Mechanics and Informatics, VNU University of Science, \\ 334 Nguyen Trai, Thanh Xuan, Hanoi, Vietnam \\ ${ }^{2}$ Faculty of Natural Science, Tran Quoc Tuan University, Son Tay, Hanoi, Vietnam
}

Received 26 June 2020

Accepted 11 July 2020

\begin{abstract}
In this paper we study linear stochastic implicit difference equations (LSIDEs for short) of index-1. We give a definition of solution and introduce an index-1 concept for these equations. The mean square stability of LSIDEs is studied by using the method of solution evaluation. An example is given to illustrate the obtained results.
\end{abstract}

Keywords: LSIDEs, index, solution, mean square stability.

\section{Introduction}

In this paper, we consider the linear time-varying stochastic implicit difference equation of the form

$$
A_{n} X(n+1)=B_{n} X(n)+C_{n} X(n) \omega_{n+1}, \quad n \in \mathbb{N},
$$

where $A_{n}, B_{n}, C_{n} \in \mathbb{R}^{d \times d}$, the leading coefficient $A_{n}$ may be singular and $\left\{\omega_{n}\right\}$ is a standard onedimensional scalar random process.

LSIDEs is generalization of linear stochastic difference equations, which have been well investigated in the literature, see [1-4]. They arise as mathematical models in various fields such as population dynamics, economics, electronic circuit systems or multibody mechanism systems with random noise (see, e.g. [5-8]. They can also be obtained from stochastic differential algebraic equations (SDAEs) by some discretization methods, see [9-12]. In comparison with linear stochastic

\footnotetext{
*Corresponding author.

Email address: nghson80@gmail.com
}

https//doi.org/ 10.25073/2588-1124/vnumap.4570 
difference equations, LSIDEs present at least two major difficulties: the first lies in the fact that it is not possible to establish general existence and uniqueness results, due to their more complicate structure; the second one is that LSIDEs need to the consistence of initial conditions and random noise.

The aim of this paper is to perform the investigation of LSIDEs. The most important qualitative properties of LSIDEs are solvability and stability. To study that, the index notion, which plays a key role in the qualitative theory of LSIDEs, should be taken into consideration in the unique solvability and the stability analysis, (see, $[6,13,14]$ ). Motivated by the index-1 concept for SDAEs in $[10,11]$, in this paper we will derive the index-1 concept for SIDEs. By using this index notion, we can establish the explicit expression of solution. After that, we shall establish the necessary conditions for the mean square stability of LSIDEs by using the method of solution evaluation.

The paper is organized as follows. In Section 2, we summarize some preliminary results of matrix analysis. In Section 3, we study solvability and stability of solution of SIDEs of index-1. The last section gives some conclusions.

\section{Preliminaries}

Let $\left(A_{n}, A_{n-1}, B_{n}\right) \in \mathbb{R}^{d \times d} \times \mathbb{R}^{d \times d} \times \mathbb{R}^{d \times d} \quad$ be a triple of matrices. Suppose that $\operatorname{rank} A_{n}=\operatorname{rank} A_{n-1}=r$ and let $T_{n} \in G L\left(\mathbb{R}^{d}\right)$ such that $\left.T_{n}\right|_{\text {ker } A_{n}}$ is an isomorphism between $\operatorname{ker} A_{n}$ and $\operatorname{ker} A_{n-1}$, put $A_{-1}=A_{0}$. We can give such an operator $T_{n}$ by the following way: let $Q_{n}$ (resp. $Q_{n-1}$ ) be a projector onto $\operatorname{ker} A_{n}$ (resp. onto $\operatorname{ker} A_{n-1}$ ); find the non-singular matrices $V_{n}$ and $V_{n-1}$ such that $Q_{n}=V_{n} Q_{n}^{(0)} V_{n}^{-1}$ and $Q_{n-1}=V_{n-1} Q_{n-1}^{(0)} V_{n-1}^{-1}$ where $Q_{n}^{(0)}=\operatorname{diag}\left(0, \mathrm{I}_{d-r}\right)$ and finally we obtain $T_{n}$ by putting $T_{n}=V_{n-1} V_{n}^{-1}$.

Now, we introduce sub-spaces and matrices

$$
\begin{aligned}
& S_{n}:=\left\{z \in \mathbb{R}^{d}: B_{n} z \in i m A_{n}\right\}, n \in \mathbb{N}, \\
& G_{n}:=A_{n}-B_{n} T_{n} Q_{n}, P_{n}:=I-Q_{n}, \\
& Q_{n-1}:=-T_{n} Q_{n} G_{n}^{-1} B_{n}, \tilde{P}_{n-1}:=I-Q_{n-1} .
\end{aligned}
$$

We have the following lemmas, see [15-17].

Lemma 2.1. The following assertions are equivalent

a) $S_{n} \cap \operatorname{ker} A_{n-1}=\{0\}$;

b) The matrix $G_{n}=A_{n}-B_{n} T_{n} Q_{n}$ is non-singular;

c) $\mathbb{R}^{d}=S_{n} \oplus \operatorname{ker} A_{n-1}$.

Lemma 2.2. Suppose that the matrix $G_{n}$ is non-singular. Then, there hold the following relations:

i) $P_{n}=G_{n}^{-1} A_{n}$, where $P_{n}=I-Q_{n}$;

ii) $-G_{n}^{-1} B_{n} T_{n} Q_{n}=Q_{n}$; 
iii) $Q_{n-1}$ is the projector onto $\operatorname{ker} A_{n-1}$ along $S_{n}$;

iv) $P_{n} G_{n}^{-1} B_{n}=P_{n} G_{n}^{-1} B_{n} P_{n-1}, Q_{n} G_{n}^{-1} B_{n}=Q_{n} G_{n}^{-1} B_{n} P_{n-1}-T_{n}^{-1} Q_{n-1}$;

v) $T_{n} Q_{n} G_{n}^{-1}$ does not depend on the choice of $T_{n}$ and $Q_{n}$.

Finally, let $\{\Omega, F, \mathrm{P}\}$ be a basic probability space, $F_{n} \in F, n \in \mathbb{N}$, be a family of $\sigma-$ algebraic, $\mathrm{E}$ be an expectation, $\left\{\omega_{n}\right\}: \omega_{n} \in \mathbb{R}$ be a sequence of mutually independent $F_{n}$-adapted random variables and independent on $F_{k}, k<n$ satisfying $\mathrm{E} \omega_{n}=0, \mathrm{E} \omega^{2}=1$ for all $n \in \mathbb{N}$.

\section{Main results}

Let us consider the linear stochastic implicit difference equations (LSIDEs)

$$
A_{n} X(\mathrm{n}+1)=B_{n} X(\mathrm{n})+C_{n} X(\mathrm{n}) \omega_{n+1}, n \in \mathbb{N},
$$

with the initial condition $X(0)=P_{-1} X_{0}$ where $A_{n}, B_{n}, C_{n} \in \mathbb{R}^{d \times d}$ with $\operatorname{rank} A_{n}=r<d$ and $\left\{\omega_{n}\right\}: \omega_{n} \in \mathbb{R}$ is a sequence of mutually independent $F_{n}-$ adapted random variables and independent on $F_{k}, k<n$ satisfying $\mathrm{E} \omega_{n}=0, \mathrm{E} \omega^{2}=1$ for all $n \in \mathbb{N}$. The homogeneous equation associated to (3.1) is

$$
A_{n} X(\mathrm{n}+1)=B_{n} X(\mathrm{n}), n \in \mathbb{N} .
$$

Definition 3.1. A stochastic process $\{X(n)\}$ is said to be a solution of the SIDE (3.1) if with probability $1, X(\mathrm{n})$ satisfies (3.1) for all $n \in \mathbb{N}$ and $X(\mathrm{n})$ is $F_{n}$-measurable.

Now, we give an index-1 concept for LSIDEs.

Definition 3.2. The LSIDE (3.1) called tractable with index-1 (or for short, of index-1) if

(i) $\operatorname{rank} A_{n}=r=\mathrm{constant;}$

(ii) $\operatorname{ker} A_{n-1} \cap S_{n}=\{0\}$;

(iii) im $C_{n} \subset$ im $A_{n}$ for all $n \in \mathbb{N}$.

Remark 1. The conditions $(i)$ and $(i i)$ are used for the index-1 concept for implicit difference equations, see [15-17]. This natural restriction (iii) is the so-called condition that the noise sources do not appear in the constraints, or equivalently a requirement that the constraint part of solution process is not directly affected by random noise which is motivated by the index-1 concept for SDAEs (see, e.g. $[10,11])$.

By using the above notion, we solve the problem of existence and uniqueness of solution of (3.1) in the following theorem.

Theorem 3.3. If equation (3.1) is of index-1, then for any $n \in \mathbb{N}$ and with the initial condition $X(0)=\tilde{P}_{-1} X_{0}$, it admits a unique solution $X(\mathrm{n})$ which given by the formula 


$$
X(\mathrm{n})=\tilde{P}_{n-1} u(\mathrm{n}),
$$

where $\left\{u_{n}\right\}$ is a sequence of $F_{n}$ - adapted random variables defined by the equation

$$
u(n+1)=P_{n} G_{n}^{-1} B_{n} u(n)+P_{n} G_{n}^{-1} C_{n} \tilde{P}_{n-1} u(n) \omega_{n+1}, n \in \mathbb{N} .
$$

Proof. Since $G_{n}^{-1} A_{n}=P_{n}, P_{n} G_{n}^{-1} A_{n}=P_{n}$ and $Q_{n} G_{n}^{-1} A_{n}=0$. Therefore, multiplying both sides of equation (3.1) by $P_{n} G_{n}^{-1}$ and $Q_{n} G_{n}^{-1}$ we get

$$
\left\{\begin{array}{l}
P_{n} X(n+1)=P_{n} G_{n}^{-1} B_{n} X(n)+P_{n} G_{n}^{-1} C_{n} X(n) \omega_{n+1}, \\
0=Q_{n} G_{n}^{-1} B_{n} X(n)+Q_{n} G_{n}^{-1} C_{n} X(n) \omega_{n+1} .
\end{array}\right.
$$

Since equation (3.1) is of index-1, im $C_{n} \subset \operatorname{im} A_{n}$ and hence $Q_{n} G_{n}^{-1} C_{n}=0$. Then the above equation is reduced to

$$
\left\{\begin{array}{l}
P_{n} X(n+1)=P_{n} G_{n}^{-1} B_{n} X(n)+P_{n} G_{n}^{-1} C_{n} X(n) \omega_{n+1}, \\
0=Q_{n} G_{n}^{-1} B_{n} X(n) .
\end{array}\right.
$$

On the other hand, by item iv) of Lemma 2.2, we have

$$
P_{n} G_{n}^{-1} B_{n}=P_{n} G_{n}^{-1} B_{n} P_{n-1}, \mathrm{Q}_{n} G_{n}^{-1} B_{n}=Q_{n} G_{n}^{-1} B_{n} P_{n-1}-T_{n}^{-1} Q_{n-1} .
$$

Therefore, (3.4) is equivalent to

$$
\left\{\begin{array}{l}
P_{n} X(n+1)=P_{n} G_{n}^{-1} B_{n} P_{n-1} X(n)+P_{n} G_{n}^{-1} C_{n} X(n) \omega_{n+1}, \\
\left(Q_{n} G_{n}^{-1} B_{n} P_{n-1}-T_{n}^{-1} Q_{n-1}\right) X(n)=0
\end{array}\right.
$$

or equivalently,

$$
\left\{\begin{array}{l}
P_{n} X(n+1)=P_{n} G_{n}^{-1} B_{n} P_{n-1} X(n)+P_{n} G_{n}^{-1} C_{n} X(n) \omega_{n+1}, \\
Q_{n-1} X(n)=T_{n} Q_{n} G_{n}^{-1} B_{n} P_{n-1} X(n) .
\end{array}\right.
$$

Putting $u(n)=P_{n-1} X(n), v(\mathrm{n})=\mathrm{Q}_{n-1} X(n)$, we imply that $v(n)=-\tilde{Q}_{n-1} u(\mathrm{n})$ and

$$
\begin{aligned}
X(n) & =P_{n-1} X(n)+Q_{n-1} X(n)=u(n)+v(n) \\
& =u(n)-\tilde{Q}_{n-1} u(n)=\left(I-\tilde{Q}_{n-1}\right) u(n)=\tilde{P}_{n-1} u(n) .
\end{aligned}
$$

Therefore, equation (3.5) becomes

$$
\left\{\begin{array}{l}
u(n+1)=P_{n} G_{n}^{-1} B_{n} u(n)+P_{n} G_{n}^{-1} C_{n} \tilde{P}_{n-1} u(n) \omega_{n+1}, \\
v(n)=-\tilde{Q}_{n-1} u(n) .
\end{array}\right.
$$

The first equation of (3.7) is an explicit stochastic difference equation. For a given initial condition $u(0)$, this equation determines the unique solution $u(n)$ which is $F_{n}-$ measurable. This implies that $v(n)=-\tilde{Q}_{n-1} u(n)$ and $X(n)=\tilde{P}_{n-1} u(n)$ are so. Thus, with the consistent initial condition 
$X(0)=\tilde{P}_{-1} X_{0}$, equation (3.1) have a unique solution $X(n)$ which is given by formulas (3.6), (3.7). The proof is complete.

Now, we study stability of the SIDE (3.1) of index-1. First, we introduce the following stability notion.

Definition 3.4. The trivial solution of equation (3.1) is called:

- Mean square stable if for any $\varepsilon>0$ and there exists a $\delta>0$ such that $\mathrm{E}\|X(n)\|^{2}<\varepsilon, \forall n \in \mathbb{N}$, if $\mathrm{E}\left\|P_{-1} X(0)\right\|^{2}<\delta$.

- Asymptotically mean square stable if it is mean square stable and with $\mathrm{E}\left\|P_{-1} X(0)\right\|^{2}<\infty$ the solution $X(n)$ of (3.1) satisfies $\lim _{n \rightarrow \infty} \mathrm{E}\|X(n)\|^{2}=0$.

If the trivial solution of equation (3.1) is mean square stable (resp. asymptotically mean square stable) then we say equation (3.1) is mean square stable (resp. asymptotically mean square stable).

Theorem 3.5. Assume that $K_{1}:=\sup _{n \geq 0}\left\|\tilde{P}_{n-1}\right\|<\infty$. Then if there exists a positive sequence $\left\{\alpha_{n}\right\}$ with $K_{2}:=\sum_{n=0}^{\infty} \alpha_{n}<\infty$ such that

$$
\left\|P_{n} G_{n}^{-1} B_{n}\right\|^{2}+\left\|P_{n} G_{n}^{-1} C_{n} \tilde{P}_{n-1}\right\|^{2} \leq 1+\alpha_{n}, \forall n \geq 0,
$$

then equation (3.1) is mean square stable. If there exists a positive sequence $\left\{\beta_{n}\right\}$ with $\sum_{n=0}^{\infty} \beta_{n}=\infty$ such that

$$
\left\|P_{n} G_{n}^{-1} B_{n}\right\|^{2}+\left\|P_{n} G_{n}^{-1} C_{n} \tilde{P}_{n-1}\right\|^{2} \leq 1-\beta_{n}, \forall n \geq 0,
$$

then equation (3.1) is asymptotically mean square stable.

Proof. We have

$$
\begin{aligned}
\|u(n+1)\|^{2}= & \left\|P_{n} G_{n}^{-1} B_{n} u(n)+P_{n} G_{n}^{-1} C_{n} X(n) \omega_{n+1}\right\|^{2} \\
= & \left\langle P_{n} G_{n}^{-1} B_{n} u(n)+P_{n} G_{n}^{-1} C_{n} X(n) \omega_{n+1}, P_{n} G_{n}^{-1} B_{n} u(n)+P_{n} G_{n}^{-1} C_{n} X(n) \omega_{n+1}\right\rangle \\
= & \left\langle P_{n} G_{n}^{-1} B_{n} u(n), P_{n} G_{n}^{-1} B_{n} u(n)\right\rangle+2\left\langle P_{n} G_{n}^{-1} B_{n} u(n), P_{n} G_{n}^{-1} C_{n} X(n) \omega_{n+1}\right\rangle \\
& \quad+\left\langle P_{n} G_{n}^{-1} C_{n} X(n) \omega_{n+1}, P_{n} G_{n}^{-1} C_{n} X(n) \omega_{n+1}\right\rangle \\
= & \left\|P_{n} G_{n}^{-1} B_{n} u(n)\right\|^{2}+2\left\langle P_{n} G_{n}^{-1} B_{n} u(n), P_{n} G_{n}^{-1} C_{n} X(n)\right\rangle \omega_{n+1} \\
& \quad+\left\|P_{n} G_{n}^{-1} C_{n} X(n)\right\|^{2} \omega_{n+1}^{2} .
\end{aligned}
$$

Since $\omega_{n+1}$ is independent on $F_{n}$, it follows that

$$
\mathrm{E}\left(\left\langle P_{n} G_{n}^{-1} B_{n} u(n), P_{n} G_{n}^{-1} C_{n} X(n)\right\rangle \omega_{n+1}\right)=\mathrm{E}\left\langle P_{n} G_{n}^{-1} B_{n} u(n), P_{n} G_{n}^{-1} C_{n} X(n)\right\rangle \mathrm{E} \omega_{n+1}=0 .
$$




$$
\begin{aligned}
\mathrm{E}\left(\left\|P_{n} G_{n}^{-1} C_{n} X(n)\right\|^{2} \omega_{n+1}^{2}\right) & =\mathrm{E}\left\|P_{n} G_{n}^{-1} C_{n} X(n)\right\|^{2} \mathrm{E} \omega_{n+1}^{2} \\
& =\mathrm{E}\left\|P_{n} G_{n}^{-1} C_{n} X(n)\right\|^{2}=\mathrm{E}\left\|P_{n} G_{n}^{-1} C_{n} \tilde{P}_{n-1}(n)\right\|^{2} .
\end{aligned}
$$

Therefore,

$$
\begin{aligned}
\mathrm{E}\|u(n+1)\|^{2} & =\mathrm{E}\left\|P_{n} G_{n}^{-1} B_{n} u(n)\right\|^{2}+\mathrm{E}\left\|P_{n} G_{n}^{-1} C_{n} \tilde{P}_{n-1} u(n)\right\|^{2} \\
& \leq\left(\left\|P_{n} G_{n}^{-1} B_{n}\right\|^{2}+\left\|P_{n} G_{n}^{-1} C_{n} \tilde{P}_{n-1}\right\|^{2}\right) \mathrm{E}\|u(n)\|^{2} .
\end{aligned}
$$

If $\left\|P_{n} G_{n}^{-1} B_{n}\right\|^{2}+\left\|P_{n} G_{n}^{-1} C_{n} \tilde{P}_{n-1}\right\|^{2} \leq 1+\alpha_{n}$ then

$$
\mathrm{E}\|u(n+1)\|^{2} \leq\left(1+\alpha_{n}\right) \mathrm{E}\|u(n)\|^{2} \leq e^{\alpha_{n}} \mathrm{E}\|u(n)\|^{2}, \forall n \geq 0 .
$$

By induction, we get

$$
\mathrm{E}\|u(n)\|^{2} \leq e^{\sum_{k=0}^{n-1} \alpha_{k}} \mathrm{E}\|u(0)\|^{2} \leq e^{K_{2}} \mathrm{E}\|u(0)\|^{2} .
$$

This implies that $\mathrm{E}\|X(n)\|^{2}=\mathrm{E}\left\|\tilde{P}_{n-1} u(n)\right\|^{2} \leq K_{1}^{2} e^{K_{2}} \mathrm{E}\|u(0)\|^{2}$. Therefore, by the definition, equation (3.1) is mean square stable. Similarly, if $\left\|P_{n} G_{n}^{-1} B_{n}\right\|^{2}+\left\|P_{n} G_{n}^{-1} C_{n} \tilde{P}_{n-1}\right\|^{2} \leq 1-\beta_{n}$ then we get

$$
\mathrm{E}\|X(n)\|^{2} \leq K_{1}^{2} e^{-\sum_{k=0}^{n-1} \beta_{k}} \mathrm{E}\|u(0)\|^{2}
$$

Since $\sum_{n=0}^{\infty} \beta_{n}=\infty, \lim _{n \rightarrow \infty} \mathrm{E}\|X(n)\|^{2}=0$ and hence equation (3.1) is asymptotically mean square stable. The theorem is proved.

Now consider the LSIDE with constant coefficient

$$
A X(n+1)=B X(n)+C X(n) \omega_{n+1}, n \in \mathbb{N},
$$

where $A, B, C \in \mathbb{R}^{d \times d}$ and $\left\{\omega_{n}\right\}: \omega_{n} \in \mathbb{R}$ is a sequence of mutually independent $F_{n}$-adapted random variables and independent on $F_{k}, k<n$ satisfying $\mathrm{E} \omega_{n}=0, \mathrm{E} \omega_{n}^{2}=1$ for all $n \in \mathbb{N}$. Note that the pair $(A, B)$ of index-1 can be transformed to Weierstraß-Kronecker canonical form, i.e., there exist nonsingular matrices $W, U \in \mathbb{R}^{d \times d}$ such that

$$
A=W\left(\begin{array}{cc}
I_{r} & 0 \\
0 & 0
\end{array}\right) U^{-1}, \mathrm{~B}=\mathrm{W}\left(\begin{array}{cc}
J & 0 \\
0 & I_{n-r}
\end{array}\right) U^{-1}
$$

where $I_{r}, I_{n-r}$ are identity matrices of indicated size, $J \in \mathbb{R}^{r \times r}$ (see, e.g. $[13,18]$ ). Then, we have

$$
P_{n}=\tilde{P}_{n}=P=U\left(\begin{array}{cc}
I_{r} & 0 \\
0 & 0
\end{array}\right) U^{-1}, \mathrm{Q}_{n}=\tilde{Q}_{n}=Q=U\left(\begin{array}{cc}
0 & 0 \\
0 & I_{n-r}
\end{array}\right) U^{-1},
$$




$$
G=A-B Q=W\left(\begin{array}{cc}
I_{r} & 0 \\
0 & -I_{n-r}
\end{array}\right) U^{-1}
$$

Corollary 3.6. Assume that equation (3.8) has index-1. Then, if $\left\|P G^{-1} B\right\|^{2}+\left\|P G^{-1} C P\right\|^{2} \leq 1$ then equation (3.1) is mean square stable. If $\left\|P G^{-1} B\right\|^{2}+\left\|P G^{-1} C P\right\|^{2}<1$ then equation (3.8) is asymptotically mean square stable.

Example 3.7. Consider the LSIDE with constant coefficient (3.8) with

$$
A=\left(\begin{array}{ll}
2 & 0 \\
1 & 0
\end{array}\right), \mathrm{B}=\left(\begin{array}{cc}
1 & 1 \\
\frac{1}{2} & 2
\end{array}\right), C=\left(\begin{array}{cc}
\frac{1}{2} & 1 \\
0 & 0
\end{array}\right)
$$

Then, it is easy to see that $P=\left(\begin{array}{ll}1 & 0 \\ 0 & 0\end{array}\right), G=\left(\begin{array}{ll}2 & -1 \\ 1 & -2\end{array}\right)$ and we obtain

$$
\left\|P G^{-1} B\right\|^{2}+\left\|P G^{-1} C P\right\|^{2}=\frac{5}{6}<1 .
$$

Thus, this equation is asymptotically mean square stable.

\section{Conclusion}

In this paper, we have investigated linear stochastic implicit difference equations (LSIDEs). The index-1 concept for these equations has been derived. After that we have established the explicit expression of solution. Finally, characterizations of the mean square stability for LSIDEs are given by the method of solution evaluation.

\section{Acknowledgments}

This work was supported by NAFOSTED project 101.01-2017.302.

\section{References}

[1] L. Shaikhet, Lyapunov functionals and stability of stochastic difference equations, Springer-Verlag London, 2011.

[2] L. Shaikhet, Necessary and Sufficient Conditions of Asymptotic Mean Square Stability for Stochastic Linear Difference Equations, Applied Mathematics Letters 10 (3) (1997) 111-115.

[3] F. Ma, T.K. Caughey, Mean stability of stochastic difference systems, International Journal of Non-Linear Mechanics 17 (2) (1982) 69--84.

[4] Z. Yang, D. Xu, Mean square exponential stability of impulsive stochastic difference equations, Applied Mathematics Letters 20 (2007) 938--945.

[5] T. Brüll, Existence and uniqueness of solutions of linear variable coefficient discrete-time descriptor systems, Linear Algebra Appl. 431 (2009) 247-265. 
[6] V.H. Linh, N.T.T. Nga and D.D. Thuan, Exponential stability and robust stability for linear time-varying singular systems of second-order difference equations, SIAM J. Matrix Anal. Appl. 39 (2018) 204-233.

[7] D.G. Luenberger, Dynamic equations in descriptor form", IEEE Trans. Automat. Control 22 (1977) 312-322.

[8] D.D. Thuan, N.H. Son, Stochastic implicit difference equations of index-1, preprint 2019, submitted for publication.

[9] L. Brancik, E. Kolarova, Application of Stochastic Differential-Algebraic Equations in Hybrid MTL Systems Analysis, Elektronika ir Elektrotechnika 20 (2014) 41-45.

[10] N.D. Cong, N.T. The, Stochastic differential - algebraic equations of index-1, Vietnam J. Math. 38 (1) (2010), 117-131.

[11] R. Winkler, Stochastic differential algebraic equations of index 1 and applications in circuit simulation, Journal of Computational and Applied Mathematics 157 (2003) 477--505.

[12] O. Scheina, G. Denk, Numerical solution of stochastic differential-algebraic equations with applications to transient noise simulation of microelectronic circuits, Journal of Computational and Applied Mathematics 100 (1998) 77--92.

[13] P. Kunkel, V. Mehrmann, Differential - algebraic equations, analysis and numerical solution, EMS Publishing House, Zürich, 2006.

[14] R. Lamour, R. März, C. Tischendorf, Differential-algebraic equations: a projector based analysis. Springer, Berlin, 2013.

[15] P.K. Anh, N.H. Du, L.C. Loi, Singular difference equations: an overview, Vietnam J. Math. 35 (2007), pp. 339 372.

[16] P.K. Anh, P.T. Linh, Stability of periodically switched discrete-time linear singular systems, Journal of Difference Equations and Applications 23 (2017) 1680-1693.

[17] N.H. Du, T.K. Duy and V.T. Viet, Degenrate cocycle index-1 and lyapunov exponents, Stochastics and Dynamics 7 (2) (2007) 229-245.

[18] N.H. Du, V.H. Linh, V. Mehrmann, D.D. Thuan, Stability and robust stability of linear time-invariant delay differential-algebraic equations, SIAM J. Matrix Anal. Appl. 34 (2013) 1631-1654. 